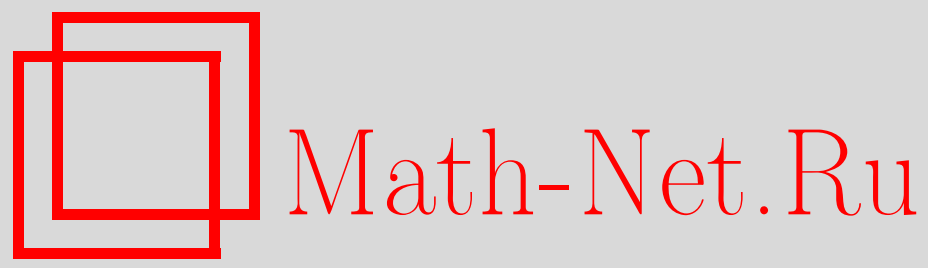

М. В. Павлов, С. И. Свинолупов, Р. А. Шарипов, Инвариантный критерий гидродинамической интегрируемости, Функи. анализ и его прил., 1996, том 30, выпуск 1, 18-29

DOI: https://doi.org/10.4213/faa478

Использование Общероссийского математического портала MathNet.Ru подразумевает, что вы прочитали и согласны с пользовательским соглашением

http://www . mathnet.ru/rus/agreement

Параметры загрузки:

IP : 3.81 .55 .215

26 апреля 2023 г., 12:52:21

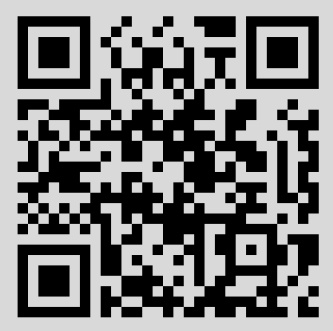


Функииональный анализ и его приложения

1996, т. 30, вып. 1, с. 18-29

УдК 517.9

\section{Инвариантный критерий гидродинамической интегрируемости}

(с) 1996. М. В. Павлов, С. И. СвинолуПов, Р. А. ШАРипов ${ }^{1}$

\section{§1. Введение}

Системы квазилинейных уравнений в частных производных первого порядка возникают при описании различных процессов динамики сплошных сред. Специальный подкласс таких систем известен как класс систем гидродинамического muna. В пространственно одномерном случае эти системы наиболее изучены. Они имеют вид

$$
u_{t}^{i}=\sum_{j=1}^{n} A_{j}^{i}(\mathbf{u}) u_{x}^{j}, \quad i=1, \ldots, n .
$$

Среди систем (1.1) выделяется подкласс систем, обладающих инвариантами Римана. Это системы, которые обратимыми преобразованиями

$$
\tilde{u}^{i}=\tilde{u}^{i}\left(u^{1}, \ldots, u^{n}\right), \quad i=1, \ldots, n,
$$

приводятся к диагональному виду

$$
u_{t}^{i}=\lambda_{i}(\mathbf{u}) u_{x}^{i}, \quad i=1, \ldots, n
$$

(суммирования по $i$ нет). Система уравнений (1.1) называется гидродинамически интегрируемой, если она обладает континуальным набором гидродинамических симметрий (или гидродинамических законов сохранения), который параметризуется $n$ произвольными функциями одной переменной. Для диагональных систем (1.3), у которых характеристические скорости $\lambda_{i}$ попарно различны, имеется хорошо развитая дифференциально-геометрическая теория интегрирования (см. обзоры $[1,2]$ ). Как показано в [2], приведенная к инвариантам Римана система уравнений (1.3) гидродинамически интегрируема тогда и только тогда, когда для нее выполнено условие

$$
\partial_{i}\left(\frac{\partial_{j} \lambda_{k}}{\lambda_{j}-\lambda_{k}}\right)=\partial_{j}\left(\frac{\partial_{i} \lambda_{k}}{\lambda_{i}-\lambda_{k}}\right), \quad i \neq k \neq j,
$$

где $\partial_{i}=\partial / \partial u^{i}$ и $\partial_{j}=\partial / \partial u^{j}$. Условие (1.4) в литературе известно как условие полугамильтоновости. В случае выполнения условия (1.4) диагональная система уравнений гидродинамического типа (1.3) интегрируется «обобщенным методом годографа» (подробности см. в работе [2]).

${ }^{1}$ Работа выполнена при финансовой поддержке Фонда Сороса (ISF), проекты RK4000, MLY000, и РФФИ, проекты 93-011-16088, 93-01-00273. 
В $\S 2$ данной работы мы рассматриваем вопрос о гидродинамической интегрируемости для систем уравнений вида (1.1) с матрицей скоростей $A_{j}^{i}(\mathbf{u})$ общего положения, собственные значения которой попарно различны. Для них удается установить следующий факт: всякая гидродинамически интегрируемая система уравнений (1.1) с матрицей скоростей общего положения диагонализуема ${ }^{2}$. Объединяя этот факт с результатами [2], можно сформулировать следующую теорему.

Теорема 1. Система уравнений (1.1) с матричей общего положения гидродинамически интегрируема тогда и только тогда, когда она диагонализуема и полугамильтонова.

Проверка интегрируемости уравнений (1.1) общего положения, согласно теореме 1 , должна включать три этапа:

(1) проверку факта диагонализуемости,

(2) фактическую диагонализацию преобразованием (1.2),

(3) проверку условия полугамильтоновости (1.4).

Первый этап этой процедуры осуществляется с помощью инвариантного дифференциально-геометрического критерия диагонализуемости из [3]. Он состоит в проверке тождественного зануления некоторого тензора (тензора Хантиеса), который строится по матрице скоростей $A_{j}^{i}(\mathbf{u})$. K системам уравнений (1.1) этот критерий диагонализуемости был впервые применен в [4].

Следующий этап фактической диагонализации состоит в нахождении собственных чисел и собственных векторов матрицы $A_{j}^{i}(\mathbf{u})$, в выборе нормировки этих векторов и в решении некоторой системы обыкновенных дифференциальных уравнений. Этот этап вносит наибольшую степень неэффективности во всю процедуру, ибо найти решение системы дифференциальных уравнений явно удается далеко не всегда. В случае эффективного преодоления второго этапа третий (проверка соотношений (1.4)) содержит лишь вычислительные трудности.

Наличие неэффективного второго этапа в изложенной выше процедуре проверки интегрируемости уравнений (1.1) связано с отсутствием инвариантного критерия гидродинамической интегрируемости для этих уравнений. Основная цель данной работы - преодоление этого существенного недостатка. Как отмечалось в [1], еще Риман заметил, что теория систем вида (1.1) - это теория тензоров, так как при обратимых преобразованиях (1.2) компоненты матрицы $A_{j}^{i}(\mathbf{u})$ меняются по тензорному закону. Поэтому естественно было ожидать, что и условие (1.4) может быть записано в инвариантном тензорном виде. В 44 данной работы по матрице скоростей $A_{j}^{i}(\mathbf{u})$ построен тензор, равенство нулю которого эквивалентно выполнению условий (1.4). Таким образом, получен инвариантный критерий гидродинамической интегрируемости для систем (1.1) общего положения. Процедура проверки гидродинамической интегрируемости таких систем уравнений становится полностью эффективной и включает два этапа:

(1) проверку зануления тензора Хантиеса,

(2) проверку зануления тензора гидродинамической интегрируемости.

${ }^{2}$ Возможно, этот результат не является новым, однако нам не удалось найти его в литературе. 
Для построения тензора гидродинамической интегрируемости мы используем дифференциально-геометрический аппарат, предложенный Фрёлихером и Нейенхейсом в [5] и [6]. Он представлен в $\S 3$. Теория Фрёлихера-Нейенхейса связывает с каждым дифференцируемым многообразием некоторую супералгебру Ли тензорных полей типа $(1, p)$ (см. [7]).

Авторы выражают благодарность В. Э. Адлеру и И. Ю. Черданцеву за плодотворные дискуссии и Б. И. Сулейманову за интерес к работе.

\section{§2. Гидродинамическая интегрируемость}

Рассмотрим систему уравнений гидродинамического типа (1.1) и дополним ее еще одной системой уравнений гидродинамического типа с динамикой по переменной $\tau$

$$
u_{\tau}^{i}=\sum_{j=1}^{n} B_{j}^{i}(\mathbf{u}) u_{x}^{j}, \quad i=1, \ldots, n .
$$

ОПРедЕлЕниЕ 1. Система уравнений (2.1) называется гидродинамической симметрией системы уравнений (1.1), если уравнения (1.1) и (2.1) совместны.

Непосредственным вычислением можно убедиться в том, что условие совместности систем (1.1) и (2.1) записывается в следующей бескоординатной форме:

$$
\begin{gathered}
\mathbf{A B}=\mathbf{B A}, \\
{[\mathbf{A X}, \mathbf{B X}]-\mathbf{A}[\mathbf{X}, \mathbf{B X}]-\mathbf{B}[\mathbf{A X}, \mathbf{X}]=0,}
\end{gathered}
$$

где $\mathbf{X}$ - произвольное векторное поле.

Пусть операторное поле $\mathbf{A}=\mathbf{A}(\mathbf{u})$ из (1.1) имеет $n$ попарно различных собственных значений $\lambda_{i}=\lambda_{i}(\mathbf{u})$. Через $\mathbf{X}_{1}, \ldots, \mathbf{X}_{n}$ обозначим репер, заданный собственными векторами линейного оператора $\mathbf{A}$. Выбор собственных векторов неоднозначен, имеется калибровочная свобода в $n$ скалярных множителей:

$$
\mathbf{X}_{i}(\mathbf{u}) \rightarrow f_{i}(\mathbf{u}) \mathbf{X}_{i}(\mathbf{u}), \quad f_{i} \neq 0, i=1, \ldots, n .
$$

Для сокращения записи введем следующие обозначения для производных Ли вдоль векторных полей $\mathbf{X}_{1}, \ldots, \mathbf{X}_{n}$ :

$$
L_{i}=L_{\mathbf{X}_{i}} .
$$

Попарные коммутаторы векторных полей $\mathbf{X}_{1}, \ldots, \mathbf{X}_{n}$ удобно разложить по реперу, образованному этими полями:

$$
L_{i} \mathbf{X}_{j}=\left[\mathbf{X}_{i}, \mathbf{X}_{j}\right]=\sum_{k=1}^{n} c_{i j}^{k} \mathbf{X}_{k}
$$

Параметры $c_{i j}^{k}=c_{i j}^{k}(\mathbf{u})$ естественно назвать структурными скалярами репера $\mathbf{X}_{1}, \ldots, \mathbf{X}_{n}$. Термин структурные константы для $c_{i j}^{k}$ не подходит, ибо эти величины зависят от точки $\mathbf{u}$.

Из алгебры хорошо известно, что перестановочность матрищы В с матрицей $\mathbf{A}$, имеющей попарно различные собственные числа, означает одновременную диагонализуемость матриц $\mathbf{A}$ и $\mathbf{B}$ в репере $\mathbf{X}_{1}, \ldots, \mathbf{X}_{n}$. Поэтому всякий 
линейный оператор $\mathbf{B}$, удовлетворяющий соотношению (2.2), полностью определяется заданием собственных значений $\mu_{i}=\mu_{i}(\mathbf{u})$. Заметим, что из (2.3) вытекает следующее соотношение, выполненное для любых двух векторных полей $\mathbf{X}$ и $\mathbf{Y :}$

$[\mathbf{A X}, \mathbf{B Y}]+[\mathbf{A Y}, \mathbf{B X}]-\mathbf{A}[\mathbf{X}, \mathbf{B Y}]-\mathbf{A}[\mathbf{Y}, \mathbf{B X}]-\mathbf{B}[\mathbf{A X}, \mathbf{Y}]-\mathbf{B}[\mathbf{A Y}, \mathbf{X}]=0$

Подставим $\mathbf{X}=\mathbf{X}_{i}$ и $\mathbf{Y}=\mathbf{X}_{j}$ в это соотношение и получим, что оно эквивалентно двум наборам соотношений. Первый алгебраичен относительно собственных чисел $\mu_{i}$ матрицы $\mathbf{B}$ :

$$
c_{j k}^{i}\left(\lambda_{j}-\lambda_{k}\right) \mu_{i}+c_{j k}^{i}\left(\lambda_{k}-\lambda_{i}\right) \mu_{j}+c_{j k}^{i}\left(\lambda_{i}-\lambda_{j}\right) \mu_{k}=0, \quad i \neq j, j \neq k, k \neq i .
$$

Второй набор содержит дифференциальные уравнения в частных производных относительно $\mu_{i}$

$$
L_{i} \mu_{j}=\lambda_{i j} \frac{\mu_{i}-\mu_{j}}{\lambda_{i}-\lambda_{j}}, \quad i \neq j .
$$

Здесь и далее для краткости используются обозначения $\lambda_{i j}=L_{i} \lambda_{j}$ в терминах производных Ли (2.5).

Система дифференциальных уравнений (2.8) является переопределенной. В случае ее совместности максимальная степень произвола в ее решениях составляет $n$ функций одной переменной. Пусть такая степень произвола фактически реализовалась. Тогда

$$
\mu_{i}=\mu_{i}\left(f_{1}, \ldots, f_{n}, \mathbf{u}\right) .
$$

Подставим (2.9) в (2.7). Это приводит к возникновению функциональных связей между параметрами $f_{1}\left(z_{1}\right), \ldots, f_{n}\left(z_{n}\right)$ из $(2.9)$, что противоречит их произвольности. Следовательно, максимальный произвол в решениях уравнений (2.8) достигается лишь при условии тривиальности соотношений (2.7). Это условие приводит к следующему соотношению для структурных скаляров, определенных согласно соотношению (2.6):

$$
c_{i j}^{k}=0, \quad i \neq j, j \neq k, k \neq i .
$$

Лемма 1. Для того чтобы линейный оператор $\mathbf{A}=\mathbf{A}(\mathbf{u})$ обиего положения был диагонализуем точечным преобразованием (1.2), необходимо и достаточно, чтобы выполнялись соотночения (2.10).

Приведем набросок доказательства этой леммы. Оператор $\mathbf{A}(\mathbf{u})$ диагонализуется в репере из своих собственных векторов. Для диагонализуемости преобразованием (1.2) этот репер должен быть координатным репером, т. е. он должен иметь нулевые структурные скаляры, $c_{i j}^{k}=0$. Условие $(2.10)$ обеспечивает зануление большей части структурных скаляров. Зануления остальных можно добиться за счет калибровочной свободы из (2.4).

В силу леммы 1 дальнейший анализ становится излишним. Для диагональных систем уравнений (1.3) анализ условий совместности уравнений (2.8) был проделан С. П. Царевым в работе [2]. В недиагональном случае в дополнение к (2.10) 
мы получим следующие соотношения:

$$
L_{i}\left(\frac{\lambda_{j k}}{\lambda_{j}-\lambda_{k}}\right)-L_{j}\left(\frac{\lambda_{i k}}{\lambda_{i}-\lambda_{k}}\right)+\frac{c_{j i}^{j} \lambda_{j k}}{\lambda_{j}-\lambda_{k}}-\frac{c_{i j}^{i} \lambda_{i k}}{\lambda_{i}-\lambda_{k}}=0,
$$

выполненные при $i \neq j, j \neq k, k \neq i$. Соотношения (2.11) представляют собой запись условий полугамильтоновости (1.4) в репере из собственных векторов оператора $\mathbf{A}(\mathbf{u})$.

Теорема 2. Система уравнений (1.1) с матрицей скоростей общего положения обладает континуальньм набором гидродинамических симметрий $c$ функциональным произволом в $n$ функций одной переменной тогда и только тогда, когда она диагонализуема и полугамильтонова.

Теперь изучим вопрос о гидродинамических законах сохранения. На множестве решений системы уравнений (1.1) определим интегральный функционал следующего вида:

$$
F=\int f(\mathbf{u}) d x
$$

называемый гидродинамическим законом сохранения или первым интегралом для уравнений (1.1), если $\dot{F}=0$ в силу этих уравнений. Нетрудно проверить, что интегральный функционал (2.12) есть гидродинамический закон сохранения для системы уравнений (1.1) тогда и только тогда, когда при любом выборе векторных полей $\mathbf{X}$ и $\mathbf{Y}$ выполнено соотношение

$$
\left(L_{\mathbf{X}} L_{\mathbf{A Y}}-L_{\mathbf{Y}} L_{\mathbf{A X}}-L_{\mathbf{A}[\mathbf{X}, \mathbf{Y}]}\right) f=0 .
$$

Соотношение (2.13) представляет собой систему дифференциальных уравнений относительно неизвестной функции $f(\mathbf{u})$, которую необходимо исследовать на совместность. Введем обозначение $L_{i} f=\varphi_{i}$. Подставив в соотношение $(2.13)$ вместо векторных полей $\mathbf{X}$ и $\mathbf{Y}$ векторные поля из репера $\mathbf{X}_{1}, \ldots, \mathbf{X}_{n}$, получаем систему уравнений вида

$$
L_{i} \varphi_{j}=\sum_{k=1}^{n} B_{i j}^{k} \varphi_{k}, \quad i \neq j,
$$

где набор функций $B_{i j}^{k}$ определяется формулами

$$
B_{i j}^{k}=c_{i j}^{k} \frac{\lambda_{k}-\lambda_{i}}{\lambda_{j}-\lambda_{i}}+\frac{\lambda_{j i} \delta_{i}^{k}}{\lambda_{j}-\lambda_{i}}-\frac{\lambda_{i j} \delta_{j}^{k}}{\lambda_{j}-\lambda_{i}} .
$$

Уравнения (2.14) аналогичны уравнениям (2.8). В случае совместности их решения имеют произвол не более чем в $n$ функций одной переменной. Пусть такая степень произвола реализовалась. Найдем дифференциальные следствия уравнений (2.14). Среди них имеются соотношения

$$
B_{j k}^{i} L_{i} \varphi_{i}-B_{i k}^{j} L_{j} \varphi_{j}-c_{i j}^{k} L_{k} \varphi_{k}=-\sum_{q=1}^{n} R_{k i j}^{q} \varphi_{q},
$$


выполненные при $i \neq j, j \neq k, k \neq i$. Величины $R_{k i j}^{q}$ вычисляются для $i \neq j$, $j \neq k, k \neq i$ по следующей формуле:

$$
R_{k i j}^{q}=L_{i} B_{j k}^{q}+\sum_{s \neq i}^{n} B_{i s}^{q} B_{j k}^{s}-L_{j} B_{i k}^{q}-\sum_{s \neq j}^{n} B_{j s}^{q} B_{i k}^{s}-\sum_{s \neq k}^{n} c_{i j}^{s} B_{s k}^{q} .
$$

Величины $L_{i} \varphi_{i}, L_{j} \varphi_{j}$ и $L_{k} \varphi_{k}$ не определяются уравнениями $(2.14)$, чем и объясняется произвол в $n$ функций для решений этих уравнений. Соотношения же (2.16) в случае, когда они нетривиальны, устанавливают функциональную связь между этими величинами, сокращая степень функционального произвола. Поэтому в случае максимального произвола в решениях (2.14) имеем

$$
B_{j k}^{i}=B_{i k}^{j}=c_{i j}^{k}=0, \quad i \neq j, j \neq k, k \neq i .
$$

Из (2.18) в силу леммы 1 вытекает диагонализуемость оператора $\mathbf{A}(\mathbf{u})$ точечным преобразованием (1.2). В силу (2.15) уравнения (2.14) в этом случае записываются в следующей форме:

$$
L_{i} \varphi_{j}=-\frac{\lambda_{j i} \varphi_{i}-\lambda_{i j} \varphi_{j}}{\lambda_{i}-\lambda_{j}}+c_{i j}^{j} \varphi_{j}, \quad i \neq j .
$$

Условие совместности уравнений (2.19) определяется величинами (2.17) следующим образом: $R_{k i j}^{q}=0$. При учете (2.18) это условие в точности совпадает с (2.11). Несмотря на то что уравнения (2.8) и (2.19) различны, их условия совместности совпадают и определяют условие полугамильтоновости в репере из собственных векторов оператора $\mathbf{A}(\mathbf{u})$. Сказанное выше доказывает теорему 1 в еще одной формулировке.

Теорема 3. Система уравнений (1.1) с матрицей скоростей общего положения обладает континуальным набором гидродинамических законов сохранения с функииональным произволом в $n$ функиий одной переменной тогда и только тогда, когда она диагонализуема и полугамильтонова.

\section{§3. Скобка Фрёлихера-Нейенхейса и супералгебра Ли векторнозначных дифференциальных форм}

Пусть $\mathbf{A}$ - тензорное поле типа $(1, p)$, кососимметричное по ковариантным компонентам. Оно определяет векторнозначную $p$-форму $\mathbf{A}=\mathbf{A}\left(\mathbf{X}_{1}, \ldots, \mathbf{X}_{p}\right)$. Здесь $\mathbf{X}_{1}, \ldots, \mathbf{X}_{p}$ - произвольные векторные поля. Пусть $\mathbf{B}-$ второе тензорное поле типа $(1, q)$, определяющее вторую векторнозначную форму $\mathbf{B}\left(\mathbf{X}_{1}, \ldots, \mathbf{X}_{q}\right)$. Назовем тензорные поля $\mathbf{A}$ и $\mathbf{B}$ тензорными полями рана 1 , если они представляются в следующем виде:

$$
\mathbf{A}=\mathbf{a} \otimes \alpha, \quad \mathbf{B}=\mathbf{b} \otimes \beta,
$$

где $\mathbf{a}$ и $\mathbf{b}$ - векторные поля, a $\alpha$ и $\beta$ - дифференциальные формы. Для тензорных полей вида (3.1) определим спаривание $\{\mathbf{A}, \mathbf{B}\}$ (скобку ФрёлихераНейенхейса) по следующей формуле:

$$
\begin{array}{r}
\{\mathbf{A}, \mathbf{B}\}=[\mathbf{a}, \mathbf{b}] \otimes \alpha \wedge \beta-\mathbf{a} \otimes L_{\mathbf{b}} \alpha \wedge \beta+\mathbf{b} \otimes \alpha \wedge L_{\mathbf{a}} \beta \\
+(-1)^{p} \mathbf{a} \otimes \iota_{\mathbf{b}} \alpha \wedge d \beta+(-1)^{p} \mathbf{b} \otimes d \alpha \wedge \iota_{\mathbf{a}} \beta .
\end{array}
$$


Через $\iota_{\mathbf{a}}$ и $\iota_{\mathbf{b}}$ в формуле (3.2) обозначены дифференцирования подстановки. Для $r$-формы $\omega$ и векторного поля с выражение $\iota_{\mathbf{c}} \omega$ есть $(r-1)$-форма вида

$$
\iota_{\mathbf{c}} \omega\left(\mathbf{X}_{1}, \ldots, \mathbf{X}_{r-1}\right)=r \omega\left(\mathbf{c}, \mathbf{X}_{\mathbf{1}}, \ldots, \mathbf{X}_{\mathbf{r}-\mathbf{1}}\right) .
$$

Операция $\iota_{\mathbf{c}}$ называется также внутренним произведением относительно векторного поля с (см. [8]).

Теорема 4. Скобка $\{\mathbf{A}, \mathbf{B}\}$, определенная для тензорных полей $\mathbf{A} и \mathbf{B}$ ранга 1 соотношением (3.2), однозначно продолжается на случай произвольных тензорных полей типа $(1, p)$ u $(1, q)$, кососимметричных по ковариантным компонентам.

ДокаЗАтельство. Всякое тензорное поле А типа $(1, p)$ может быть разложено в сумму тензорных полей ранга 1 следующим образом:

$$
\mathbf{A}=\sum_{i} \mathbf{A}_{i}=\sum_{i} \mathbf{a}_{i} \otimes \alpha_{i}
$$

Аналогичное разложение имеет место и для тензорного поля В. Поэтому скобку (3.2) для произвольных $\mathbf{A}$ и $\mathbf{B}$ можно определить так:

$$
\{\mathbf{A}, \mathbf{B}\}=\sum_{i} \sum_{j}\left\{\mathbf{A}_{i}, \mathbf{B}_{j}\right\} .
$$

Однако разложение (3.3) неоднозначно. Поэтому должна быть проверена корректность определения $\{\mathbf{A}, \mathbf{B}\}$ посредством (3.4). Степень неоднозначности разложения (3.3) для $\mathbf{A}$ определяется следующими определяющими тождествами в тензорной алгебре:

$$
\begin{aligned}
(\mathbf{a}+\widetilde{\mathbf{a}}) \otimes \alpha & =\mathbf{a} \otimes \alpha+\widetilde{\mathbf{a}} \otimes \alpha, \\
\mathbf{a} \otimes(\alpha+\widetilde{\alpha}) & =\mathbf{a} \otimes \alpha+\mathbf{a} \otimes \widetilde{\alpha}, \\
(f \mathbf{a}) \otimes \alpha & =\mathbf{a} \otimes(f \alpha),
\end{aligned}
$$

где $f$ - произвольное скалярное поле. Неоднозначность, вызванная тождествами (3.5), не влияет на величину скобки (3.4), ибо соотношение (3.2) аддитивно по а и $\alpha$. Покажем, что неоднозначность, вызванная тождеством (3.6), также не сказывается на величине $\{\mathbf{A}, \mathbf{B}\}$. Для этого вычислим скобку по формуле (3.2) сначала для $\mathbf{A}=(f \mathbf{a}) \otimes \alpha$, а затем для $\mathbf{A}=\mathbf{a} \otimes(f \alpha)$, после чего сравним результаты. Вычисления базируются на следующих формулах (см. [8]):

$$
\begin{gathered}
{[f \mathbf{a}, \mathbf{b}]=f[\mathbf{a}, \mathbf{b}]-\mathbf{a} L_{\mathbf{b}} f, \quad L_{f \mathbf{a}} \beta=f L_{\mathbf{a}} \beta+d f \wedge \iota_{\mathbf{a}} \beta,} \\
L_{\mathbf{b}}(f \alpha)=f L_{\mathbf{b}} \alpha+L_{\mathbf{b}} f \alpha, \quad \iota_{f} \mathbf{a} \beta=f \iota_{\mathbf{a}} \beta .
\end{gathered}
$$

Эти вычисления достаточно стандартны, и мы их не приводим. Теорема доказана.

Отметим, что при $p=q=0$ скобка (2.2) совпадает с обычным коммутатором векторных полей. При других значениях $p$ и $q$ алгебраические свойства этой скобки даются следующей теоремой. 
Теорема 5. Скобка $\{\mathbf{A}, \mathbf{B}\}$, определенная для тензорных полей $\mathbf{A} и \mathbf{B}$ ранга 1 соотношением (3.2), удовлетворяет соотношениям

$$
\begin{gathered}
\{\mathbf{A}, \mathbf{B}\}+(-1)^{p q}\{\mathbf{B}, \mathbf{A}\}=0, \\
\{\{\mathbf{A}, \mathbf{B}\}, \mathbf{C}\}(-1)^{r p}+\{\{\mathbf{B}, \mathbf{C}\}, \mathbf{A}\}(-1)^{p q}+\{\{\mathbf{C}, \mathbf{A}\}, \mathbf{B}\}(-1)^{q r}=0,
\end{gathered}
$$

в силу которых она определяет структуру градуированной супералгебры Ли в тензорных полах типа $(1, m)$, кососимметричных по ковариантным компонентам.

Пусть $\mathbf{A}$ и $\mathbf{B}$ - тензорные поля типа $(1,1)$ (операторнозначные поля). Тензорное поле $\mathbf{S}=2\{\mathbf{A}, \mathbf{B}\}$ есть векторнозначная 2-форма. Ее значения на векторных полях $\mathbf{X}$ и $\mathbf{Y}$ можно записать в виде

$$
\begin{aligned}
\mathbf{S}(\mathbf{X}, \mathbf{Y})=[\mathbf{A X}, \mathbf{B Y}]+[\mathbf{B X}, \mathbf{A Y}]+\mathbf{A B}[\mathbf{X}, \mathbf{Y}]+\mathbf{B A}[\mathbf{X}, \mathbf{Y}] \\
\\
-\mathbf{A}[\mathbf{X}, \mathbf{B Y}]-\mathbf{A}[\mathbf{B X}, \mathbf{Y}]-\mathbf{B}[\mathbf{X}, \mathbf{A Y}]-\mathbf{B}[\mathbf{A X}, \mathbf{Y}] .
\end{aligned}
$$

Тензор $\mathbf{S}$ известен как кручение Нейенхейса операторнозначных полей $\mathbf{A}$ и $\mathbf{B}$ (см. [5, 6-8]).

\section{§4. Построение тензора полугамильтоновости}

Прежде всего напомним классический результат об инвариантном критерии диагонализуемости операторного поля $\mathbf{A}$, который уже упоминался в $\S 1$ (см. [3], а также [4] и [6]). Рассмотрим частный случай тензора (3.7)

$$
\mathbf{N}=\{\mathbf{A}, \mathbf{A}\} .
$$

Его обычно называют тензором Нейенхейса. В силу (3.7) для него имеем

$$
\mathbf{N}(\mathbf{X}, \mathbf{Y})=[\mathbf{A X}, \mathbf{A Y}]+\mathbf{A}^{2}[\mathbf{X}, \mathbf{Y}]-\mathbf{A}[\mathbf{X}, \mathbf{A Y}]-\mathbf{A}[\mathbf{A X}, \mathbf{Y}] .
$$

Тензор Хантиеса определяется по тензору Нейенхейса (4.1), согласно следующей формуле:

$$
\mathbf{H}(\mathbf{X}, \mathbf{Y})=\mathbf{N}(\mathbf{A X}, \mathbf{A Y})+\mathbf{A}^{2} \mathbf{N}(\mathbf{X}, \mathbf{Y})-\mathbf{A N}(\mathbf{X}, \mathbf{A Y})-\mathbf{A N}(\mathbf{A X}, \mathbf{Y}) .
$$

Он имеет тот же тип, что и тензор $\mathbf{N}$, и тоже является векторнозначной кососимметрической 2-формой.

Теорема 6 (критерий диагонализуемости). Оператор $\mathbf{A}(\mathbf{u})$ общего положения с попарно различными собственными числами диагонализуется точечныц преобразованием (1.2) тогда и только тогда, когда построенныи й по нему тензор Хантиеса (4.2) тождественно равен нулю.

Критерий диагонализуемости в форме этой теоремы был доказан Хантиесом в работе [3] и использован применительно к системам гидродинамического типа (1.1) в работе [4].

ДокАЗАТЕЛЬСТво. В силу кососимметричности билинейной формы (4.2) ее равенство нулю достаточно проверять на векторных полях $\mathbf{X}=\mathbf{X}_{i}$ и $\mathbf{Y}=\mathbf{X}_{j}$ из репера собственных векторов оператора $\mathbf{A}(\mathbf{u})$ при $i \neq j$. Непосредственным 
вычислением получаем

$$
\mathbf{H}\left(\mathbf{X}_{i}, \mathbf{X}_{j}\right)=\sum_{k=1}^{n}\left(\lambda_{i}-\lambda_{k}\right)^{2}\left(\lambda_{j}-\lambda_{k}\right)^{2} c_{i j}^{k} \mathbf{X}_{k}
$$

В силу (4.3) равенство $\mathbf{H}\left(\mathbf{X}_{i}, \mathbf{X}_{j}\right)=0$ эквивалентно выполнению соотношений (2.10). Остается лишь применить лемму 1. Критерий доказан.

Практическое применение этого критерия для выяснения факта диагонализуемости основано на следующих формулах для вычисления компонент тензоров $\mathbf{N}$ и $\mathbf{H}$ через компоненты матрицы скоростей $\mathbf{A}(\mathbf{u})$ в произвольной системе координат:

$$
\begin{aligned}
N_{i j}^{k} & =\sum_{s=1}^{n}\left(A_{i}^{s} \partial_{s} A_{j}^{k}-A_{j}^{s} \partial_{s} A_{i}^{k}+A_{s}^{k} \partial_{j} A_{i}^{s}-A_{s}^{k} \partial_{i} A_{j}^{s}\right), \\
H_{i j}^{k} & =\sum_{s=1}^{n} \sum_{r=1}^{n}\left(A_{s}^{k} A_{r}^{s} N_{i j}^{r}-A_{s}^{k} N_{r j}^{s} A_{i}^{r}-A_{s}^{k} N_{i r}^{s} A_{j}^{r}+N_{s r}^{k} A_{i}^{s} A_{j}^{r}\right) .
\end{aligned}
$$

Пусть В - операторнозначное тензорное поле типа (1.1), и пусть $\mathbf{Q}$ - некоторое кососимметричное по ковариантным компонентам тензорное поле типа $(1,2)$. Обозначим через $\mathbf{K}$ скобку Фрёлихера-Нейенхейса этих двух полей, $\mathbf{K}=3\{\mathbf{Q}, \mathbf{B}\}$. Для векторнозначной 3-формы, определяемой полем $\mathbf{K}$, имеем

$$
\begin{aligned}
\mathbf{K}(\mathbf{X}, \mathbf{Y}, \mathbf{Z})=\mathbf{B}[\mathbf{X}, \mathbf{Q}(\mathbf{Y}, \mathbf{Z})]-[\mathbf{B X}, \mathbf{Q}(\mathbf{Y}, \mathbf{Z})] \\
+\mathbf{B Q}(\mathbf{X},[\mathbf{Y}, \mathbf{Z}])+\mathbf{Q}(\mathbf{X}, \mathbf{B}[\mathbf{Y}, \mathbf{Z}]) \\
-\mathbf{Q}(\mathbf{X},[\mathbf{B Y}, \mathbf{Z}])-\mathbf{Q}(\mathbf{X},[\mathbf{Y}, \mathbf{B Z}])+\ldots
\end{aligned}
$$

Многоточием в (4.6) обозначены еще 12 слагаемых, которые получаются из выписанных циклической перестановкой $\mathbf{X}, \mathbf{Y}$ и $\mathbf{Z}$. Переходя к выводу условия полугамильтоновости в инвариантной форме, отметим, что всюду выше (см. теоремы 1, 3 и 5) это условие фигурирует в совокупности с условием диагонализуемости для матриц скоростей общего положения. Никакой отдельной роли в рамках изложенной выше теории оно не играет. Поэтому схема дальнейших действий следующая: мы построим тензор, условие равенства нулю которого после приведения матрицы $\mathbf{A}(\mathbf{u})$ к инвариантам Римана принимает вид (1.4). Само соотношение (1.4) рационально по $\lambda_{i}$. Перепишем его в полиномиальном виде. Для этого определим следующие величины

$$
\begin{aligned}
\alpha_{k i j}^{k}=- & \left(\lambda_{i}-\lambda_{j}\right)\left(\lambda_{i}-\lambda_{k}\right)\left(\lambda_{j}-\lambda_{k}\right) \partial_{i j} \lambda_{k} \\
& -\left(\lambda_{i}-\lambda_{j}\right)\left(\lambda_{i}+\lambda_{j}-2 \lambda_{k}\right) \partial_{i} \lambda_{k} \partial_{j} \lambda_{k} \\
& +\left(\lambda_{i}-\lambda_{k}\right)^{2} \partial_{i} \lambda_{j} \partial_{j} \lambda_{k}-\left(\lambda_{j}-\lambda_{k}\right)^{2} \partial_{j} \lambda_{i} \partial_{i} \lambda_{k}
\end{aligned}
$$

Условие полугамильтоновости (1.4) записывается в виде условия равенства нулю введенных величин (4.7), а именно

$$
\alpha_{k i j}^{k}=0, \quad i \neq k \neq j .
$$

Частные производные в (4.7), как и в (1.4), берутся по переменным $u^{1}, \ldots, u^{n}$, в которых матрица скоростей системы уравнений (1.1) диагональна. Репер 
$\mathbf{X}^{1}, \ldots, \mathbf{X}^{n}$ собственных векторов матрицы $\mathbf{A}$ выберем совпадающим с координатным репером в этих переменных.

Используя скобку Фрёлихера-Нейенхейса, из тензора скоростей $\mathbf{A}$ образуем тензор $\mathbf{K}$, определив его следующей формулой:

$$
\mathbf{K}=3\left\{\{\mathbf{A}, \mathbf{A}\}, \mathbf{A}^{2}\right\}=3\left\{\mathbf{N}, \mathbf{A}^{2}\right\} .
$$

Тензор $\mathbf{K}$ определяет векторнозначную 3-форму. Значения этой формы на векторах репера $\mathbf{X}^{1}, \ldots, \mathbf{X}^{n}$ могут быть вычислены по формуле (4.6). В результате такого вычисления получаем

$$
\mathbf{K}\left(\mathbf{X}_{k}, \mathbf{X}_{i}, \mathbf{X}_{j}\right)=K_{k i j}^{k} \mathbf{X}_{k}+K_{k i j}^{i} \mathbf{X}_{i}+K_{k i j}^{j} \mathbf{X}_{j}
$$

(суммирования по $i, j, k$ нет). Нас интересует лишь одна из групп компонент тензора $\mathbf{K}$. Остальные получаются циклической перестановкой индексов $\left(K_{k i j}^{i}=K_{i j k}^{i}, K_{k i j}^{j}=K_{j k i}^{j}\right)$ из коэффициентов $K_{k i j}^{k}$ в формуле (4.10). Имеем

$K_{k i j}^{k}-\alpha_{k i j}^{k}=2\left(\lambda_{i}-\lambda_{j}\right)\left[\left(\lambda_{i}-\lambda_{k}\right)+\left(\lambda_{j}-\lambda_{k}\right)\right]\left(\partial_{i} \lambda_{k} \partial_{j} \lambda_{k}-\partial_{i} \lambda_{j} \partial_{j} \lambda_{k}-\partial_{j} \lambda_{i} \partial_{i} \lambda_{k}\right)$.

Построим новый тензор $\mathbf{M}$ из тензора $\mathbf{N}$. Определим соответствующую полилинейную форму следующим соотношением:

$$
\begin{aligned}
\mathbf{M}(\mathbf{X}, \mathbf{Y}, \mathbf{Z})=\mathbf{N}(\mathbf{X}, \mathbf{A} \mathbf{N}(\mathbf{Y}, \mathbf{Z}))+\mathbf{N}(\mathbf{A X}, \mathbf{N}(\mathbf{Y}, \mathbf{Z})) \\
-\mathbf{N}(\mathbf{N}(\mathbf{X}, \mathbf{Z}), \mathbf{A Y})+\mathbf{N}(\mathbf{N}(\mathbf{X}, \mathbf{Y}), \mathbf{A Z}) \\
-\mathbf{N}(\mathbf{X}, \mathbf{N}(\mathbf{A Y}, \mathbf{Z}))-\mathbf{N}(\mathbf{X}, \mathbf{N}(\mathbf{Y}, \mathbf{A Z}))
\end{aligned}
$$

Для тензора $\mathbf{M}$ из (4.12) в репере из собственных векторов оператора $\mathbf{A}(\mathbf{u})$ получаем

$$
\mathbf{M}\left(\mathbf{X}_{k}, \mathbf{X}_{i}, \mathbf{X}_{j}\right)=M_{k i j}^{k} \mathbf{X}_{k}+M_{k i j}^{i} \mathbf{X}_{i}+M_{k i j}^{j} \mathbf{X}_{j}
$$

причем для компонент тензора $\mathbf{M}$ из (4.13) имеем

$$
M_{k i j}^{k}=-\left(\lambda_{i}-\lambda_{j}\right)\left(\lambda_{i}-\lambda_{k}\right)\left(\lambda_{j}-\lambda_{k}\right)\left(\partial_{i} \lambda_{k} \partial_{j} \lambda_{k}-\partial_{i} \lambda_{j} \partial_{j} \lambda_{k}-\partial_{j} \lambda_{i} \partial_{i} \lambda_{k}\right) .
$$

Коэффициенты $M_{k i j}^{i}$ и $M_{k i j}^{j}$ нас в данный момент не интересуют. Сравнив (4.11) с (4.14), построим тензор $\mathbf{Q}$,

$$
\begin{aligned}
& \mathbf{Q}(\mathbf{X}, \mathbf{Y}, \mathbf{Z})=\mathbf{K}(\mathbf{A X}, \mathbf{A Y}, \mathbf{Z})-\mathbf{K}\left(\mathbf{A}^{2} \mathbf{X}, \mathbf{Y}, \mathbf{Z}\right)-\mathbf{K}(\mathbf{X}, \mathbf{A Y}, \mathbf{A Z}) \\
&+\mathbf{K}(\mathbf{A X}, \mathbf{Y}, \mathbf{A Z})+4 \mathbf{M}(\mathbf{A X}, \mathbf{Y}, \mathbf{Z}) \\
&-2 \mathbf{M}(\mathbf{X}, \mathbf{A Y}, \mathbf{Z})-2 \mathbf{M}(\mathbf{X}, \mathbf{Y}, \mathbf{A Z}) .
\end{aligned}
$$

Для компонент тензора $\mathbf{Q}$ в репере из собственных векторов матрицы скоростей $\mathbf{A}(\mathbf{u})$ получаем соотношение

$$
\mathbf{Q}\left(\mathbf{X}_{k}, \mathbf{X}_{i}, \mathbf{X}_{j}\right)=Q_{k i j}^{k} \mathbf{X}_{k}+Q_{k i j}^{i} \mathbf{X}_{i}+Q_{k i j}^{j} \mathbf{X}_{j},
$$

аналогичное (4.10) и (4.13). Интересующие нас компоненты тензора $\mathbf{Q}$ выражаются через (4.7) и имеют вид

$$
Q_{k i j}^{k}=-\left(\lambda_{i}-\lambda_{k}\right)\left(\lambda_{j}-\lambda_{k}\right) \alpha_{k i j}^{k},
$$


Теперь мы можем построить искомый тензор полугамильтоновости по следующей формуле:

$$
\begin{aligned}
\mathbf{P}(\mathbf{X}, \mathbf{Y}, \mathbf{Z})=\mathbf{A Q}(\mathbf{X}, \mathbf{A Y}, \mathbf{Z})+\mathbf{A Q}(\mathbf{X}, \mathbf{Y}, \mathbf{A Z}) \\
-\mathbf{A}^{2} \mathbf{Q}(\mathbf{X}, \mathbf{Y}, \mathbf{Z})-\mathbf{Q}(\mathbf{X}, \mathbf{A Y}, \mathbf{A Z}) .
\end{aligned}
$$

Нетрудно проверить, что на произвольных трех векторах из репера $\mathbf{X}_{1}, \ldots, \mathbf{X}_{n}$ имеет место соотношение

$$
\mathbf{P}\left(\mathbf{X}_{k}, \mathbf{X}_{i}, \mathbf{X}_{j}\right)=\left(\lambda_{i}-\lambda_{k}\right)^{2}\left(\lambda_{j}-\lambda_{k}\right)^{2} \alpha_{k i j}^{k} \mathbf{X}_{k} .
$$

В результате доказана следующая основная теорема (инвариантный критерий полугамильтоновости):

Теорема 7. Диагонализуемьй оператор общего положения $\mathbf{A}$ с попарно различными собственньми числами полугамильтонов тогда и только тогда, когда построенный по нему тензор $\mathbf{P}$ из (4.18) равен нулю тождественно.

Доказательство теоремы прямо вытекает из формул (4.8) и (4.19). Оно не требует комментариев. В заключение приведем сводку формул, позволяющих вычислять компоненты тензора полугамильтоновости $\mathbf{P}$ по матрице скоростей $\mathbf{A}(\mathbf{u})$ в произвольной системе координат:

$$
P_{k i j}^{s}=\sum_{p=1}^{n} \sum_{q=1}^{n}\left(A_{p}^{s} Q_{k q j}^{p} A_{i}^{q}+A_{p}^{s} Q_{k i q}^{p} A_{j}^{q}-A_{q}^{s} A_{p}^{q} Q_{k i j}^{p}-Q_{k p q}^{s} A_{i}^{p} A_{j}^{q}\right) .
$$

Компоненты тензора $\mathbf{Q}$, входяшие в формулу (4.20), вычисляются на базе соотношения (4.15):

$$
\begin{aligned}
Q_{k i j}^{s}= & \sum_{p=1}^{n} \sum_{q=1}^{n}\left(A_{k}^{p} K_{p q j}^{s} A_{i}^{q}+A_{k}^{p} K_{p i q}^{s} A_{j}^{q}-A_{q}^{p} A_{k}^{q} K_{p i j}^{s}-K_{k p q}^{s} A_{i}^{p} A_{j}^{q}\right) \\
& +\sum_{p=1}^{n}\left(4 A_{k}^{p} M_{p i j}^{s}-2 M_{k p j}^{s} A_{i}^{p}-2 M_{k i p}^{s} A_{j}^{p}\right) .
\end{aligned}
$$

Компоненты тензора $\mathbf{M}$ в выписанной выше формуле вычисляются на базе соотношения (4.12):

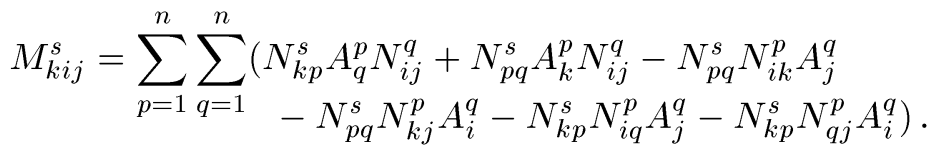

Тензор $\mathbf{K}$ вычисляется по тензору Нейенхейса с использованием скобки Фрёлихера-Нейенхейса на базе формулы (4.9). Положим $\mathbf{B}=\mathbf{A}^{2}$. Тогда для компонент тензора $\mathbf{K}$ в произвольной системе координат имеем

$$
K_{k i j}^{s}=\sum_{p=1}^{n}\left(B_{p}^{s} \partial_{k} N_{i j}^{p}-B_{k}^{p} \partial_{p} N_{i j}^{s}+N_{i j}^{p} \partial_{p} B_{k}^{s}-N_{k p}^{s} \partial_{i} B_{j}^{p}+N_{k p}^{s} \partial_{j} B_{i}^{p}\right)+\ldots
$$

Многоточием в (4.21) обозначены 10 слагаемых, которые получаются из выписанных пяти слагаемых циклической перестановкой индексов $i, j$ и $k$. 
Выписанные выше формулы, разумеется, достаточно громоздки для непосредственного счета. Однако современные компьютерные системы аналитических вычислений снимают эту проблему для любых конкретных систем уравнений гидродинамического типа, возникающих в практических приложениях.

\section{ЛитеРАтУРА}

1. Дубровин Б. А., Новиков С. П. Гидродинамика слабо деформированных солитонных решеток. Дифференциальная геометрия и гамильтонова теория. УМН, 44, вып. 6, 29-98 (1989).

2. Царев С. П. Геометрия гамильтоновых систем гидродинамического типа. Обобщенный метод годографа. Изв. АН СССР, сер. матем., 54, № 5, 10481068 (1990).

3. Haantjes $A$. On $X_{n-1}$-forming sets of eigenvectors. Indag. Math., 17, No. 2, 158$162(1955)$.

4. Ферапонтов E. В., Царев С. П. Системы гидродинамического типа, возникающие в хроматографии. Инварианты Римана и точные решения. Матем. моделирование, 3, № 2, 82-91 (1991).

5. Nijenhuis $A$. $X_{n-1}$-forming sets of eigenvectors. Indag. Math., 13, No. 2, 200-212 (1951).

6. Froelicher A., Nijenhuis A. Some new cohomology invariants for complex manifolds. Proc. Koninkl. Nederl. Akad. Wetensch. A, 59, No. 5, 540-564 (1956).

7. Березин Ф. A. Введение в алгебру и анализ с антикоммутирующими переменными. Изд-во МГУ, М. (1983).

8. Кобаяси ШІ., Номидзу К. Основы дифференциальной геометрии. Т. 1, Наука, M. (1981).

Институт теоретической физики им. Л. Д. Ландау РАН

Институт математики Уфимского отделения РАН

Башкирский университет
Поступило в редакцию 10 августа 1994 г. 